\title{
PROPAGATION OF EDGE FLAMES IN COUNTERFLOW MIXING LAYERS: EXPERIMENTS AND THEORY
}

\author{
VITO S. SANTORO, AMABLE LIÑÁN AND ALESSANDRO GOMEZ \\ Yale Center for Combustion Studies \\ Yale University \\ P.O. Box 208287 \\ New Haven, CT 06520-8287, USA
}

\begin{abstract}
Edge flames were investigated in a methane $/ \mathrm{O}_{2} / \mathrm{N}_{2}$ counterflow diffusion flame burner. In a typical experiment, a stable counterflow diffusion flame in an axysymmetric configuration was perturbed by lowering the relevant Damköhler number slightly below the extinction value, $D a_{\text {ext }}$. As a result, the flame extinguished in the vicinity of the burner axis where conditions were uniform. An edge flame extinction front quickly propagated in the radial direction, turned into an ignition edge flame, and eventually stabilized as a standing triple flame at a radial position larger than the burner radius. This sequence of events resulted from an increase of $D a$ as a function of the radial direction, consequent to a decrease in the strain rate in the radial direction. The edge flame propagation velocity in the ignition mode was measured for propagating edge flames at moderate $D a$ and for standing triple flames at large $D a$, using a combination of laser Doppler velocimetry of seeded particles, formaldehyde planar laser-induced fluorescence, and natural chemiluminescence imaging. The propagation velocity, nondimensionalized with the premixed laminar flame speed of the unburned stoichiometric mixture, was correlated with $D a$. The latter was calculated using a thermal diffusive model and velocity measurements. The nondimensional velocity reached a value of 2.6 at large $D a$, in good agreement with the estimated square root of the ratio of the unburned gas density to the burned gas density, as suggested by scaling considerations.
\end{abstract}

\section{Introduction}

Edge flames are ubiquitous in extinction and ignition processes, near the interface of oxidizer and fuel streams, or under partially premixed conditions. For example, they intervene in the stabilization of lifted non-premixed jet flames [1]. They are also relevant to regimes of turbulent combustion under large strain, when extinction holes develop in the flame, followed by reignition under rapidly fluctuating flow conditions [2]. In the ignition mode, an edge flame consists typically of a diffusion flame connected at a triple point to two premixed flames, one lean on one side, and one rich on the other. Such flames are referred to as triple flames and are characterized by positive laminar flame speeds, that is, they propagate toward the unburned mixture. In the extinction mode, the edge flames present a simple edge without any tribrachial nature, that recedes away from the unburned mixture with negative flame speed [3].

Triple flames were observed for the first time by Phillips [4] in 1965 in an experimental study in an unstrained horizontal mixing layer. Propagation speeds were also measured using particle tracking and flow visualization. The problem was dormant until it was addressed theoretically using large activation energy asymptotics [5]. After another long hiatus, this analytical approach was extended to include upstream heat conduction [6]. The issue has since remained an object of study at all levels-experimental, theoretical, and computational-and has been revisited more frequently in the past few years [7-15].

With this relatively long but spotty history, it is perhaps not too surprising that only two sets of measurements of triple flame propagation speed have been reported to date, in addition to Ref. [4]. The original experiments in Ref. [4] were revealing since they showed that the velocity at the triple point was close to the ordinary laminar flame speed of a stoichiometric mixture, and this value was a factor of two or more smaller than the velocity further upstream because of flow divergence. As a result, a triple flame has intrinsic stabilization properties, since it appears to propagate at a speed substantially higher than the ordinary laminar flame speed with respect to the unburned mixture far upstream. This fluid dynamic explanation was clarified in Ref. [7]. Ref. [3] is mostly significant for its modeling contribution and essentially repeated the same type of experiments of Ref. [4], albeit in a vertical configuration. Ref. [15] provided an extensive database of triple flame propagation in the stabilization of axisymmetric jet diffusion flames and correlated the results with curvature, mixture fraction gradient, and stretch. A recent numerical study confirmed that all these effects as well as preferential diffusion play a 
role, but flow divergence is the dominant cause for the increase of propagation speed [13].

None of these studies has examined edge flame propagation phenomena as they develop in a multiply connected turbulent flame. Counterflow diffusion flames may provide a simpler environment conceptually linked to turbulent diffusion flames via the flamelet approach. In this article, we first develop a theoretical model for the establishment of edge flames in this configuration, using the thermal-diffusive approximation. Next, we perform experiments revealing the entire phenomenology of edge flames naturally, that is, without the need for contrived geometries or peculiar arrangements and composition of the feed streams. Lastly, results for steady triple flames and for propagating ones, including detailed measurements of propagation velocity by laser Doppler velocimetry (LDV), are correlated with the relevant Damköhler number.

\section{Theoretical Framework}

If the overall chemical reaction is strongly sensitive to the temperature, two reaction regimes are possible in the mixing layer between two counterflowing jets of fuel and oxidizer. In the nearly frozen regime, mixing occurs without significant effect of the chemical reaction; in the fast burning regime the reaction is diffusion controlled, and the temperature and concentration fields can be described using the Burke-Schumann approximation of infinite chemical reaction rate. The transition between the two regimes of burning is abrupt. Extinction occurs when, with increasing strain rates, the mass burning rate of fuel per unit flame surface increases above a critical value. The process can be characterized in terms of a Damköhler number $(D a)$ that must become smaller than a critical value $D a_{\text {ext }}$ for extinction to occur [16]. Ignition occurs if a flame front is created by a localized external energy input in the chemically frozen mixing layer. This front ultimately propagates as an edge flame along the mixing layer.

The Damköhler number is one of the parameters determining the edge flame velocity $U_{\mathrm{EF}}$ relative to the frozen mixture. Other parameters include: the ratio of the unburned density to the burned value of the stoichiometric mixture, $\rho_{\mathrm{ub}} / \rho_{\mathrm{b}}[7]$; the value of the mixture fraction at stoichiometric, $Z_{s}$; and the Lewis number of the fuel, $L e_{\mathrm{F}}$ [12]. The front velocity was found to be positive only for values of the Damköhler number larger than the critical Damköhler number for extinction of the diffusion flame.

The Damköhler number $D a=1 / \chi_{s} t_{\mathrm{L}}$ is based on the scalar dissipation at the stoichiometric surface $\chi_{\mathrm{s}}$ and on a characteristic chemical time $t_{\mathrm{L}}$. The scalar dissipation $\chi_{\mathrm{s}}=D_{\mathrm{T}}|\nabla Z|_{\mathrm{s}}^{2}$ is the inverse of the characteristic mixing time and is determined by the product of the thermal diffusivity and the square of the mixture fraction gradient, evaluated at the stoichiometric surface. The inverse of the mixture fraction gradient $|\nabla Z|_{\mathrm{s}}$ is a measure of the thickness of the mixing layer $\delta_{\mathrm{m}}$. This thickness, for two counterflowing jets of characteristic radius $R_{\mathrm{O}}$ and velocity $V_{\mathrm{O}}$, is of the order $\delta_{\mathrm{m}} \cong \sqrt{D_{\mathrm{T}} R_{\mathrm{O}} / V_{\mathrm{O}}}$. Thus, $\chi_{\mathrm{s}} \cong$ $D_{\mathrm{T}} / \delta_{\mathrm{m}}^{2}$. If the Reynolds number based on $R_{\mathrm{O}}$ and $V_{\mathrm{O}}$ is large compared with unity and if the flow is laminar, $\delta_{\mathrm{m}} / R_{\mathrm{O}} \ll 1$.

The analysis will show that the thickness of the mixing layer grows, and the scalar dissipation decreases, with increasing values of $r / R_{\mathrm{O}}$. The minimum value $\delta_{\mathrm{O}}$ of $\delta_{\mathrm{m}}$ in the mixing layer, and therefore the peak value of $\chi_{\mathrm{s}}$, will be found at the center region of the mixing layer, for $r / R_{\mathrm{O}} \ll 1$. In this region, the radial velocity gradient $d u_{\mathrm{e}} / d r$ is constant and equal to one half of the axial velocity gradient, or strain rate, $A$.

The evolution of the radial velocity components at the edge of the mixing layer, $u_{\mathrm{e}}$, with $r / R_{\mathrm{O}}$, will determine the radial evolution of the stoichiometric scalar dissipation or, equivalently, of the thickness $\delta_{\mathrm{m}}$ of the mixing layer. Although variable density effects, arising mainly from the heat release associated with the diffusion flame, will slightly modify the conclusions, we shall use in the following the thermal diffusive model of constant density and transport properties for simplicity. If the two jets have the same density, $u_{\mathrm{e}}$ is the same on both sides of the mixing layer.

With the above scaling premises, we shall now consider the structure of the mixing layer imposed by the velocity field

$$
u=u_{\mathrm{e}}(r), v=-y \frac{1}{r} \frac{d}{d r}\left(r u_{\mathrm{e}}\right)
$$

$u_{\mathrm{e}}(r)=V_{\mathrm{O}} \tilde{u}\left(r / R_{\mathrm{O}}\right)$ should be obtained either experimentally (see Fig. 4 below) or by means of the inviscid analysis of the external constant density flowfield. In the Burke-Schumann limit of infinite reaction rates and for equal and constant diffusivities of mass and heat $D_{\mathrm{T}}$, the fuel and oxygen mass fractions $Y_{\mathrm{F}}$ and $Y_{\mathrm{O}}$ and the temperature $T$ can be expressed in terms of the mixture fraction $Z$. The latter is defined as

$$
Z=\frac{s Y_{\mathrm{F}}-Y_{\mathrm{O}}+Y_{\mathrm{O}, \mathrm{O}}}{s Y_{\mathrm{F}, \mathrm{F}}+Y_{\mathrm{O}, \mathrm{O}}}
$$

in terms of $s$, the oxygen mass consumed per unit mass of fuel, and the feed stream values of the fuel and oxygen mass fractions. The mass fraction $Z$ satisfies, for all values of the Damköhler number, the conservation equation

$$
u_{\mathrm{e}} \frac{\partial Z}{\partial r}+v \frac{\partial Z}{\partial y}=D_{\mathrm{T}} \frac{\partial^{2} Z}{\partial y^{2}}
$$

where $u_{\mathrm{e}}$ and $v$ are given by equation 1 and $z$ is the 
axial coordinate. Equation 3 is subject to the boundary conditions

$$
Z=0 \text { at } y \rightarrow \infty, Z=1 \text { at } y \rightarrow-\infty
$$

The constant density approximation permits a selfsimilar solution of the form

$$
Z=Z(\eta) \text { with } \eta=y / \delta_{\mathrm{m}}(r)
$$

for general forms of the velocity field $u_{\mathrm{e}}(r)$ imposed to the mixing layer. Equation 3 is satisfied if $\delta_{\mathrm{m}}(r)$ and $Z(\eta)$ are chosen so as to satisfy the equations

$$
\begin{gathered}
\left(\delta_{\mathrm{m}}^{2} / D_{\mathrm{T}}\right)\left\{u_{\mathrm{e}} \frac{1}{\delta_{\mathrm{m}}} \frac{d \delta_{\mathrm{m}}}{d r}+\frac{1}{r} \frac{d u_{\mathrm{e}}}{d r}\right\}=1 \\
\eta Z_{\eta}+Z_{\eta \eta}=0
\end{gathered}
$$

The solution of equation 7 , with the boundary conditions $Z(\infty)=0, Z(-\infty)=1$ from equation 4 , is the usual [16]

$$
Z=\frac{1}{2} \operatorname{erfc}(\eta / \sqrt{2})
$$

Thus, the mixture fraction distribution depends on $r$ through the local value $\delta_{\mathrm{m}}(r)$ of the thickness of the mixing layer. The latter is the solution of equation 6 with the symmetry condition $d \delta_{\mathrm{m}} / d r=0$ at $r$ $=0$, associated with the form of the radial velocity for $r / R_{0} \ll 1, u_{\mathrm{e}}=A r / 2$. Equation 6 can be rewritten in the form

$$
\frac{d}{d r}\left(\frac{\delta_{\mathrm{m}}^{2}}{D_{\mathrm{T}}} r^{2} u_{\mathrm{e}}^{2}\right)=2 r^{2} u_{\mathrm{e}}
$$

that can be integrated to give

$$
\delta_{\mathrm{m}}^{2} / D_{\mathrm{T}}=\left(r^{2} u_{\mathrm{e}}^{2}\right)^{-1} \int_{0}^{r} 2 r^{\prime 2} u_{\mathrm{e}}\left(r^{\prime}\right) d r^{\prime}
$$

For

$$
r / R_{\mathrm{O}} \ll 1, \delta_{\mathrm{m}}=\delta_{\mathrm{O}}=\sqrt{D_{\mathrm{T}} / A}
$$

and the characteristic diffusion time $\delta_{\mathrm{m}}^{2} / D_{\mathrm{T}}$ is constant at $1 / A$. For $r / R_{\mathrm{o}} \gg 1$ when $u_{\mathrm{e}} \rightarrow V_{\mathrm{o}}$,

$$
\delta_{\mathrm{m}} \cong\left\{D_{\mathrm{T}} \frac{2}{3} \frac{R_{\mathrm{O}}}{V_{\mathrm{O}}} \frac{r}{R_{\mathrm{O}}}\right]^{1 / 2}
$$

while the characteristic diffusion time will exhibit a linear growth as $\delta_{\mathrm{m}}^{2} / D_{\mathrm{T}}=2 r / 3 U$. The growth would be even more rapid if, because of viscous effects in the flow external to the mixing layer, $u_{\mathrm{e}}$ were to decrease below $V_{\mathrm{o}}$.

With $Z(\eta)$ given by equation 8 , the flame surface lies at $\eta=\eta_{\mathrm{s}}$ where

$$
Z=Z_{\mathrm{S}}=1 /\left(1+s \frac{Y_{\mathrm{F}, \mathrm{F}}}{Y_{\mathrm{O}, \mathrm{O}}}\right)
$$

is given by

$$
Z_{\mathrm{s}}=\frac{1}{2} \operatorname{erfc}\left(\eta_{\mathrm{s}} / \sqrt{2}\right)
$$

The non-dimensional mixture fraction gradient at $Z$ $=Z_{\mathrm{s}}$ is

$$
\left(Z_{\eta}\right)_{\mathrm{s}}=(2 \pi)^{-1 / 2} \exp \left(-\eta_{\mathrm{s}}^{2} / 2\right)
$$

and can be used to calculate the value $\chi_{\mathrm{s}}$ of the scalar dissipation at the stoichiometric surface,

$$
\chi_{\mathrm{s}}=D_{\mathrm{T}}|\nabla Z|_{\mathrm{s}}^{2}=\tilde{\chi}_{\mathrm{s}}\left(D_{\mathrm{T}} / \delta_{\mathrm{m}}^{2}\right)
$$

where

$$
\tilde{\chi}_{\mathrm{s}}=\left(Z_{\eta}\right)_{\mathrm{s}}^{2}=(1 / 2 \pi) \exp \left(-\eta_{\mathrm{s}}^{2}\right)
$$

is the non-dimensional scalar dissipation rate that does not vary along the flame surface. On the other hand, according to equation $10, \chi_{s}$ decreases with $r$ / $R_{\mathrm{o}}$ from its maximum value $A \tilde{\chi}_{\mathrm{s}}$ at the stagnation region.

Thus, the local value of the Damköhler number, $1 / \chi_{\mathrm{s}} t_{\mathrm{L}}=\delta_{\mathrm{m}}^{2} / D_{\mathrm{T}} \tilde{\chi} t_{\mathrm{L}}$, is seen to grow with $r / R_{\mathrm{o}}$ from its minimum value $1 / A \tilde{\chi}_{\mathrm{s}} t_{\mathrm{L}}$ at the stagnation point. With increasing values of the strain rate $A$, the flame will extinguish first in the stagnation region, creating an extinction front that will propagate out radially (see Fig. 1a). However, the front, with the decreasing scalar dissipation with $r$ and consequent increase of the $D a$, may become an ignition front. The front velocity would now be inward, toward the unburned mixture, and may be overcome by the outward radial flow (Fig. 1b). As a result, the edge flame will still move outward in the laboratory reference frame. Finally, at a particular radial location, the edge flame inward propagation velocity may balance the radial flow velocity $u_{\mathrm{e}}$, resulting in a stable standing edge flame, which, if $D a$ is large enough, will appear as a triple flame. In the remainder of this article, we will show evidence of this process. Once a hole has been created in the stagnation region, the flow in the diffusion flame and the concentration distributions are described by the frozen distributions [16]. Thus, flow and concentration fields that the triple flame encounters can be easily described.

\section{Experimental Setup}

An axisymmetric counterflow diffusion flame was established in the vertical burner shown in Fig. 2, with the $\mathrm{O}_{2}$ and $\mathrm{N}_{2}$ being fed from the top and $\mathrm{CH}_{4}$ and $\mathrm{N}_{2}$ from the bottom. Both sides of the burner terminated in a contraction contoured to optimize the uniformity of the axial velocity in the radial direction. The exit diameter of the two nozzles was $12.5 \mathrm{~mm}$, and the separation distance between them was kept constant at $13 \mathrm{~mm}$. Both standing and propagating edge flames were examined, the latter produced by subjecting the flame to a periodic velocity pulse generated by a loudspeaker coupled with 


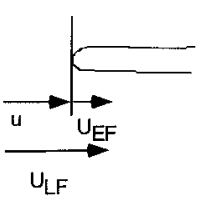

a)

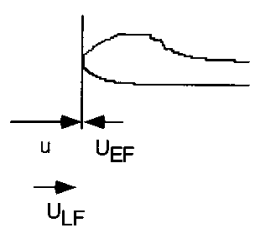

b)

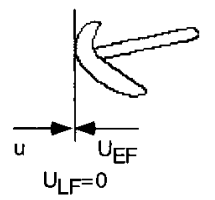

c)

FIG. 1. Sketch of typical edge flame configurations: (a) Propagating flame in extinction mode: (b) Propagating flame in ignition mode: (c) Standing triple flame. Also shown are the velocity vectors of: $u$, the radial convective flow; $U_{\mathrm{EF}}$, the edge flame propagation velocity with respect to the unburned mixture; and $U_{\mathrm{LF}}$, the velocity with respect to the laboratory reference frame.

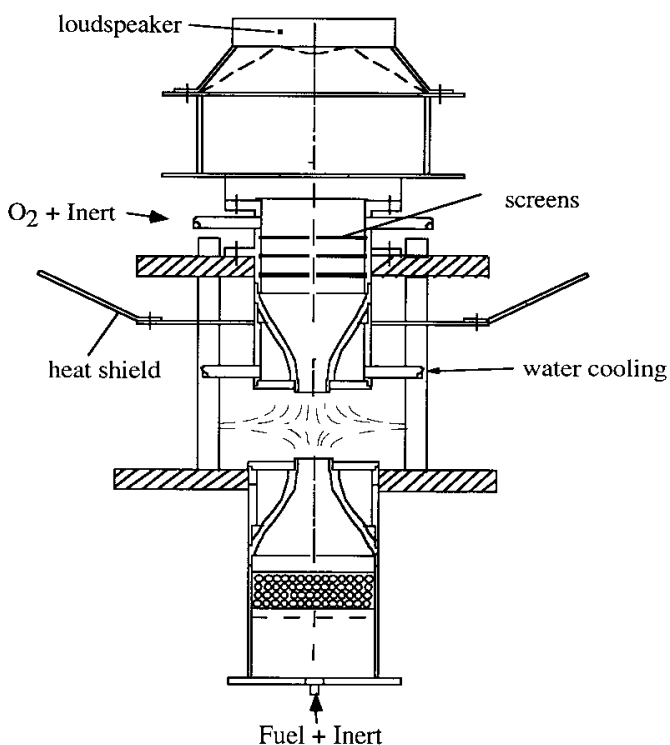

FIG. 2. Burner configuration. the top burner. The function input to the loudspeaker was designed in such a way to avoid any suction or any other perturbation of the front propagation velocity.

To monitor the edge flame, formaldehyde (HCHO) planar laser-induced fluorescence (PLIF) was used as a complementary marker of the flame. In fact, calculations under steady-state conditions, using a numerical code with detailed kinetics and transport, indicated that the peak of heat release corresponds to the location where the formaldehyde concentration precipitously drops [17]. This approach proved much easier to implement than other techniques [18]. Consequently, the third harmonic of a Nd:YAG laser, $355 \mathrm{~nm}$ and $120 \mathrm{~mJ} /$ pulse of energy, was used to excite the tail of the $4_{0}^{1}$ transition in the $\tilde{A}^{1} A_{2} \leftarrow \tilde{X}^{1} A_{1}$ band of HCHO, as in Ref. [19]. The resulting signal was detected using a gated single-stage image intensifier coupled to a CCD (Santa Barbara Instrument Group ST6B). A narrow interference filter at $415 \mathrm{~nm}$ with a full width at half maximum of $10 \mathrm{~nm}$ was used to reject flame luminescence and other interferences. Because of low signal-to-noise ratio, the fluorescence signal had to be averaged over 10 shots. Propagating edge flames could be visualized at each time of their evolution by synchronizing the CCD camera and the laser with the function generator feeding the loudspeaker, using a variable time delay.

Formaldehyde PLIF was complemented by LDV velocimetry to measure the radial velocity component in the vicinity of the triple flame and at the edge of the mixing layer. $\mathrm{Al}_{2} \mathrm{O}_{3}$ particles with a nominal diameter of $1.5 \mu \mathrm{m}$ were used for that purpose. The $95 \%$ confidence interval for the velocity measurements was estimated at \pm 0.02 of the mean. It was computed with a conservative assumption of $1 \%$ bias error (based on manufacturer specifications) and a precision limit of the mean of $1.5 \%$.

Experiments were conducted under various conditions spanning a range of strain rates from $80 \mathrm{~s}^{-1}$ to $300 \mathrm{~s}^{-1}$ for flames with different compositions, resulting in a range of the stoichiometric mixture fraction, $Z_{s}$, between 0.52 and 0.83 . In a typical experiment, a methane counterflow diffusion flame was established, and a local extinction near the flame centerline was induced. The extinction front propagated quickly outwardly in the radial direction, thereby creating a hole in the flame, similarly to Ref. [20]. As the boundary of the two impinging jets was approached, the relevant Damköhler number increased and the edge flame changed nature, turning into an ignition edge flame that eventually stabilized at a finite radius away from the centerline.

\section{Results and Discussion}

Figure 3 a shows a picture of a standing triple flame obtained by a combination of HCHO PLIF 


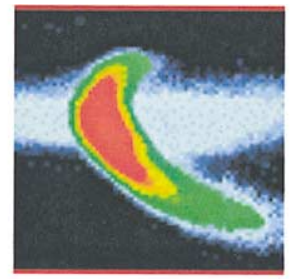

(a)

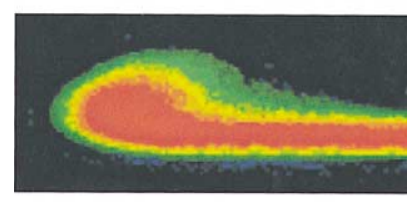

(b)

FIG. 3. (a) Standing triple flame image from HCHO PLIF (red-yellow-green) superimposed with flame chemiluminescence (light blue): (b) Propagating edge flame image from HCHO PLIF.

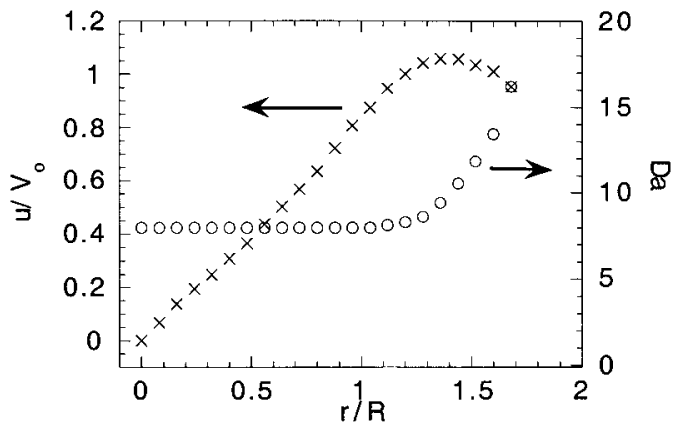

FIG. 4. Non-dimensional radial velocity (left ordinate) and Damköhler number (right ordinate) as a function of the non-dimensional radial coordinate.

and natural flame chemiluminescence. Such a flame corresponds to the sketch in Fig. 1c. The formaldehyde layer was visualized in false color and averaged over 10 shots. The intensity is highest (red) at the edge of the triple flame near the triple point, a lean side is present at the top of the image, and a more pronounced rich wing, imbedded into a lower concentration layer (green), is present at the bottom. This picture is consistent with the concentration profiles of Ref. [10]. The flame chemiluminescence is superimposed on the formaldehyde image and shows the position of the diffusion flame, appearing as an approximately horizontal light blue band. At the left of the triple flame, the chemiluminescence band is an artifact of the three-dimensionality of the flame since the flame emission is integrated over the optical path seen by the camera. Notice that the only formaldehyde on the fuel side is that associated with the premixed flame branch. As shown by additional probing at larger radial positions, formaldehyde fluorescence does reappear on the fuel side of the diffusion flame farther out, when methane resumes its primary reactant role in the diffusion flame. On the other hand, methane does not reach the trailing diffusion flame in the immediate vicinity of the triple flame, because of the divergence of the streamlines around the rich whisker at the triple point. The trailing diffusion flame is, in fact, the result of CO and $\mathrm{H}_{2}$ oxidation [9].

Figure $3 \mathrm{~b}$ shows a single shot HCHO PLIF image of a propagating edge flame corresponding to the sketch in Fig. 1b. The tribrachial nature is no longer present, possibly because the premixed flame whiskers have merged with the diffusion flame because of the relatively large local strain rate. The flame exhibits a bulge on the oxidizer side. Fig. 3b is the case in which the ignition edge flame front is swept away from the incoming convective stream. We were unable to capture the third case, the extinction edge flame (Fig. 1a), probably because the transition to an ignition edge flame occurred much too rapidly.

To assess the edge flame propagation velocity, we can revisit Fig. 1. The edge flame velocity with respect to the laboratory reference frame $U_{\mathrm{LF}}$ is the result of the competition between the convective velocity toward the front, that, in the present case, coincides with the radial component of the gaseous velocity $u(r)$ and the edge flame front velocity $U_{\mathrm{EF}}$, measured with respect to the unburned mixture and defined positive if toward that mixture (ignition front). Thus, $U_{\mathrm{LF}}=u-U_{\mathrm{EF}}$. For standing triple flames, $U_{\mathrm{LF}}=0$; hence, the radial velocity at the edge of the flame provides a direct measure of $U_{\mathrm{EF}}$. In the case of propagating fronts, these velocity measurements must be complemented by a measurement of $U_{\mathrm{LF}}$ using formaldehyde visualizations to keep track of the edge flame position in time. In particular, in this second set of experiments, the flame was extinguished by a small velocity increment imposed by the loudspeaker to a flame on the brink of extinction. The perturbation was less than $10 \%$ of the initial velocity.

Figure 4 shows the radial velocity profile (left ordinate) $u$ as measured along the radial direction in the mixing layer for standing triple flames. Left ordinate and abscissa are non-dimensionalized with respect to the axial velocity at the centerline at the outlet of the nozzle and the nozzle radius, respectively. As discussed before, for $r / R_{0}<1$, where the usual one-dimensional approximation and self-similarity apply, the profile is linear. For $r / R>1$, the 


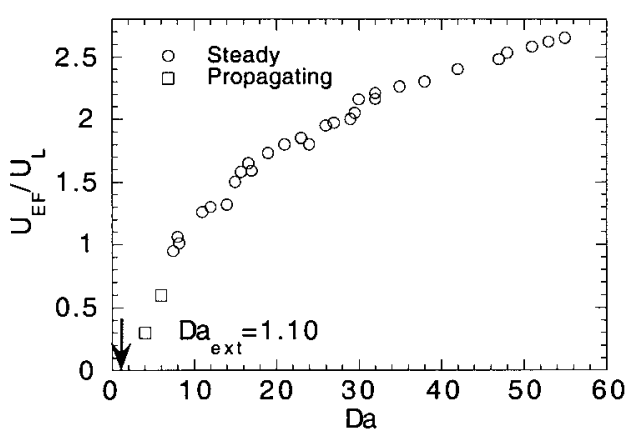

FIG. 5. Propagation velocity of the edge flame, non-dimensionalized with respect to the laminar flame speed, versus Damköhler number.

one-dimensional assumption does not hold, and the thickness of the mixing layer is not constant but depends on the radial position. In this region, a stable triple flame can be stabilized. In this particular case, the standing triple flame was positioned at $r / R_{\mathrm{o}}=$ 1.56 , and the nondimensional velocity measurement at that location is a direct measure of $U_{\mathrm{EF}}$. There is an error related to the precise determination of the upstream location where the measurements ought to be performed. It should be at the onset of the diverging streamline effect that is primarily responsible for the relative increase of triple flame propagation as compared to the ordinary laminar flame speed. Such a point is difficult to determine precisely, especially in a nonuniform flowfield as the present one. Nevertheless, the local gradients are sufficiently small to introduce an error in the measurements of $U_{\mathrm{F}}$ on the order of only $10 \%$. As a result, the measurements have good repeatability, as Fig. 5 shows.

Also shown in Fig. 4 is the local Damköhler number (right ordinate) as a function of the radial position, calculated from $D a=1 / \chi_{\mathrm{s}} t_{\mathrm{L}}$ using equation 10 . To estimate this quantity, the chemical time was evaluated using a premixed flame analogy. Namely, the chemical time can be conveniently interpreted as $t_{\mathrm{L}}=D_{\mathrm{T}} / U_{\mathrm{L}}^{2}$, that is, the residence time in the preheated layer of a planar stoichiometric premixed flame of velocity $U_{\mathrm{L}}$. In so doing, the uncertainty associated with the particular choice of chemical kinetics rate constant is removed, and this characteristic time is related to a well-defined observable. The premixed flame velocity was calculated using a onedimensional code with detailed transport properties and chemistry [21]. The thickness of the mixing layer was calculated from the integration of the radial velocity measurements at the edge of the mixing layer, and the thermal diffusivity $D_{\mathrm{T}}=k /\left(\rho c_{\mathrm{p}}\right)$ was computed calculating the thermal conductivity, $k$, the heat capacity $c_{\mathrm{p}}$, and the density $\rho$ of the relevant cold mixture of $\mathrm{CH}_{4} / \mathrm{O}_{2} / \mathrm{N}_{2}$ in stoichiometric proportions [22]. As anticipated in the theoretical section, Fig. 4 shows a constant Damköhler number as a function of the radial coordinate, where one-dimensionality and self-similarity apply. On the other hand, in the outer region this variable increases, as a result of the increase in mixing layer thickness.

The measurements of Fig. 4 can be repeated for different flow conditions, yielding different positions of the standing triple flame or different propagating edge flames. Using the procedure outlined for Fig. 4 , the $D a$ at the position of the edge flame was calculated. As a result, a correlation of edge flame velocity and $D a$ was developed. The results are presented in Fig. 5, where the edge flame velocity, non-dimensionalized with respect to the premixed flame velocity for stoichiometric composition, is plotted versus the local Damköhler number. Circles represent standing triple flames (Figs. 1c and 3a), and squares are for propagating edge flames (Figs. $1 \mathrm{~b}$ and $3 \mathrm{~b}$ ). For the latter, the front velocity was the average of the velocity calculated from the displacement of 20 images at three different positions. The radial velocity profile was measured for the same case for the steady cold flow, once the hole had already been established. Since both front velocity and radial velocity of the flow depend on the radial position, the velocity of the edge flame was based on the average value of two positions of the propagating edge flame. This measurement, inaccurate as it may be, is still useful for a qualitative understanding of edge flame dynamics. The dependence of the nondimensional edge flame velocity on the $D a$ is monotonically increasing and reaches a value of approximately 2.60. This value should be of the same order as the square root of the density ratio between the unburned mixture and burned gas [7]. A temperature measurement of the trailing diffusion flame by thin filament pyrometry, using a $15 \mu \mathrm{m} \mathrm{SiC}$ wire to minimize the perturbation of the flame, yielded a peak measurement of $1980 \mathrm{~K}$. Ratioing this value to that of the cold gas and taking the square root, neglecting molecular weight effects, we obtained a value of 2.57, in excellent agreement with the data in Fig. 5.

To understand why standing triple flames are possible, we can replot such a velocity as a function of the radial coordinate. The non-dimensional front propagation velocity results are shown in Fig. 6 together with the same radial velocity profile of Fig. 4 for a particular flame. The two curves intersect at two points, $A$ and $B$, that are both equilibrium points in the mixing layer. From a simple stability analysis, it is apparent that A is unstable and B is stable. As a result, a standing edge flame is stabilized at the outermost location. This stability condition was checked experimentally as follows. A cold mixing layer was ignited by a laser spark generated using the second harmonic of the Nd:YAG at 532, focused with a 


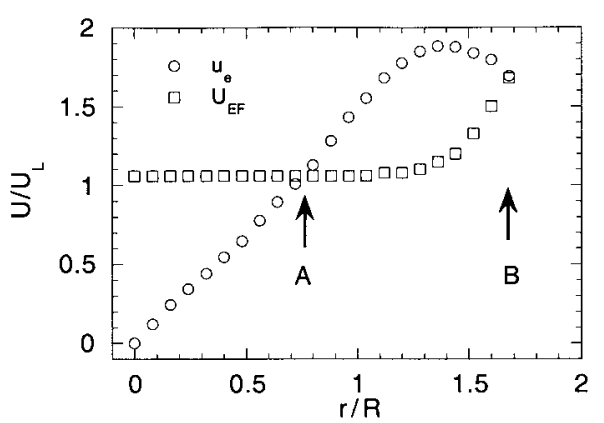

FIG. 6. Non-dimensional radial velocity and edge flame propagation velocity versus radial coordinate. Of the two intersection points, one is unstable (A) and the other is stable (B).

spherical lens $(f=12.5 \mathrm{~cm})$. Two modes of ignition were observed: an annular edge flame was generated, if the spark was localized somewhere between $0.8<r / R<2$; and a full diffusion flame (no hole) was stabilized, if the ignition source was localized between the origin and $r / R=0.8$. The boundary between these two ranges, at $r / R=0.8$, is within half a millimeter from the location of the unstable point in the mixing layer, the differences being attributable to finite spark dimensions.

\section{Conclusions}

Experiments in counterflow diffusion flames showed that the entire phenomenology of edge flames (extinction fronts, ignition fronts, tribrachial structures) can be captured, thereby mimicking the phenomenology of multiply connected turbulent flames. HCHO PLIF images of the edge flames showed tribrachial structures for standing triple flames at large $D a$ and simple edge flames at moderate $D a$. The propagation velocity of the edge flames in the ignition mode was measured using a combination of LDV techniques and HCHO PLIF. The measurements were correlated with the Damköhler number using a thermal diffusive model.

\section{Acknowledgments}

The support of NASA, under the Microgravity Science and Applications Program, grant no. NAG3-1688 (Dennis Stocker, Contract Monitor), and of NSF, grant no. CTS9904296 (Farley Fisher, Contract Monitor), is gratefully acknowledged.

\section{REFERENCES}

1. Takahashi, F., Schmoll, W. J., and Katta, V. R., Proc. Combust. Inst. 27:675-684 (1998).

2. Vervisch, L., and Poinsot, T., Annu. Rev. Fluid Mech. 30:655-91 (1998).

3. Kioni, P. N., Rogg, B., Bray, K. N. C., and Liñán, A., Combust. Flame 95:276-290 (1993).

4. Phillips, H., Proc. Combust. Inst. 10:1277-1283 (1965).

5. Liñán, A., and Crespo, A., Combust. Sci. Technol. 14:95-117 (1976).

6. Dold, J. W., Combust. Flame 76:71-88 (1989).

7. Ruetsch, G. R., Vervish, L., and Liñán, A., Phys. Fluids 7(6):1447-1454 (1995).

8. Buckmaster, J., Combust. Sci. Technol. 115:41-68 (1996).

9. Plessing, T., Terhoeven, P., Peters, N., and Mansour, M. S., Combust. Flame 115:335-353 (1998).

10. Echekki, T., and Chen, J. H., Combust. Flame 114:231-245 (1998).

11. Shay, M. L., and Ronney, P. D., Combust. Flame 112:171-180 (1998).

12. Daou, J., and Liñán, A., Combust. Theory Modelling 2:449-477 (1998).

13. Im, H. G., and Chen, J. H., Combust. Flame 119:436454 (1999).

14. Kioni, P. N., Bray, K. N. C., Greenhalgh, D. A., and Rogg, B., Combust. Flame 116:192-206 (1999).

15. Ko, Y. S., and Chung, S. H., Combust. Flame 118:151163 (1999).

16. Liñán, A., Acta Astronaut. 1:1007-1039 (1974).

17. Santoro, V. S., Kyritsis, D. C., and Gomez, A., "A Comparison of the Interaction of Laminar Vortices with Either Gaseous or Spray Counterflow Diffusion Flames" presented at the Joint Meeting of the U.S. Sections of the Combustion Institute, Washington, D.C., March 15-17, 1999.

18. Paul, P. H., and Najm, H. N., Proc. Combust. Inst. 27:45-30 (1998).

19. Harrington, J. E., and Smyth, K. C., Chem. Phys. Lett. 202:196-202 (1993).

20. Potter, A. E., Heimel, S., and Butler, J. N., Proc. Combust. Inst. 8:1027-1034 (1960).

21. Smooke, M. D., and Miller, J. A., Combust. Sci. Technol. 34:79-90 (1983).

22. Rosner, D. E., Transport Processes in Chemically Reacting Flow Systems, Butterworth-Heinemann, Stonehan, MA, 1986. 


\section{COMMENTS}

John Dold, UMIST, UK. When the Damköhler number is reduced sufficiently the speed of propagation of the flame edge becomes negative, or a front of quenching advances against the flow. Could you use your experiment to measure negative speeds of a flame edge?

Author's Reply. In this set of experiments we were not able to measure the propagation velocity of extinction fronts. The difficulty of this measurement stems from the rapid transition of these fronts to ignition fronts. Some careful redesigning of the experimental apparatus may be used in the future to circumvent this problem.

Paul Papas, Swiss Federal Institute of Technology, Switzerland. In our earlier work [1], we also reported the transition of a disk-shaped diffusion flame, after extinction, to an annular-shaped edge flame, and the possible formation of an annular-shaped edge flame upon ignition. In our methane-air experiments, the extinguishment of the diskshaped diffusion flame (located on the air-side of the stagnation plane for this case), resulted in an annular-shaped edge flame whose anchoring position shifted to the stagnation plane. Could you please comment on the location of the diffusion flame with respect to the stagnation plane (prior to transition to an annular-shaped edge flame structure) for the mixture conditions of your methane $/ \mathrm{N}_{2} / \mathrm{O}_{2}$ experiments? Also, using two-dimensional counterflow direct numerical simulations in our study, we briefly examined the influence of the overall stoichiometry on the annullar-shaped edge flame structure, the resulting edge flame shape orientation, and the existence (or not) of a diffusion flame "tail" of a tribrachial flame. Did you examine the influence of stoichiometry or mixtures strength experimentally?

\section{REFERENCE}

1. Papas, P., and Tomboulides, A. G., "Observations of Annular-Shaped Edge Flames in a Counterflow", CST communications, Vol. 1, July (2000).

See also Papas, P., and Tomboulides, A. G., "Observations of Tribrachial Flames in a Counterflow", presented at Eastern States Section of the Combustion Institute, E. Hartford, CT, Oct. 27-29 (1997).

Author's Reply. In the experimental results presented in this work both the stoichiometry and the strain rate were changed simultaneously to investigate the effect of Damköhler number on the propagation. In particular, the mixture fraction at the stoichiometric surface, $\mathrm{Zs}$, was bracketed between 0.52 and 0.83 . Thus, the flame was located always on the fuel side with respect to the gas stagnation plane.

Amable Liñán, Universidad Politecnica de Madrid, Spain. Just a comment on the variety in the structure and velocity of edge and triple flames. These depend on many parameters, such as the Lewis number of the reactants, the ratio of flame to ambient temperature, the stoichiometric mixture fraction, and the Damköhler number, or the square of the ratio of mixing layer thickness to flame thickness. Although significant work has been carried out, mainly theoretically due to the significant dependence on so many parameters, a lot remains to be done on this problem, which would be of much importance to understand the structure of lifted diffusion flames and on ignition in unpremixed systems, in laminar or turbulent flows.

Author's Reply. The comment requires no reply. 